\title{
Gastro-oesophageal reflux disease: time to re-visit
}

\author{
ES De Silva ${ }^{1}$, JDVC Lekamwasam ${ }^{2}$ \\ ${ }^{1}$ Senior Registrar in Medicine, ${ }^{2}$ Consultant Physician, Teaching Hospital, Karapitiya, Galle.
}

In 1892 William Osler suggested that oesophagus could be a source of episodic chest pain but only in 1955 that Flood et al. was able to demonstrate this [1]. He showed that oesophageal aspirate from a patient with heart burn had more acid content than the control. It is well recognized that Gastro-oesophageal Reflux Disease (GORD) could be a source of chest pain as well as regurgitation. It is a common problem in the general population but there was some uncertainty regarding diagnosis until the recent past. Although there were number of guidelines and recommendations for the diagnosis and management of GORD, a universally accepted definition of GORD was lacking. This uncertainty led to much confusion in clinical practice as well as research.

Few years back an International Consensus Group was appointed to develop a global definition and classification of GORD. This Group included 44 experts from 18 different countries. They studied the literature, data bases and came up with a set of statements on GORD which were reviewed, modified over a period of 2 years. A modified Delphi process was used to develop the final consensus definition on GORD. At the final vote, $94 \%$ of the final 51 statements were approved by $90 \%$ of the Consensus Group, and $90 \%$ of statements were accepted with strong agreement or with minor reservations. The results of the study were first presented at the World Congress of Gastroenterology in Montreal. This is known as Montreal Definition and Classification of Gastro-oesophageal Reflux Disease. The final document was endorsed by the World Organization of Gastroenterology (WGO-OMGE) as "an important development in a critical area of gastroenterology worldwide."
According to the global definition of GORD it is a condition which develops when the reflux of stomach contents causes troublesome symptoms and/or complications [2]. There are some important features in this definition. It identifies reflux of stomach contents as the etiology and symptoms should trouble an individual to constitute the diagnosis. In clinical practice, individual patient should decide whether symptoms are troublesome or not and in epidemiological studies minor symptoms occurring two days or moderate/severe symptoms occurring once a day per week is taken as troublesome. This definition also includes common complications of GORD like reflux oesophagitis, stricture, Barrett's oesophagus and adenocarcinoma of the oesophagus if they are symptomatic. Retrosternal burning pain and regurgitation had been identified as the most common symptom where as most common injury had been identified ass reflux oesophagitis. Retrosternal burning pain is defined as feeling of burning pain behind the breast bone and regurgitation as feeling of stomach content regurgitating in to the mouth or hypopharynx.

The Montreal Definition and Classification identifies GORD as a disease which could present in many different ways. This is a new concept by which GORD-related manifestations are identified as a set of syndromes. If the symptoms are related to oesophagus it is classified as Oesophageal Syndromes and if the symptoms are not related to oesophagus then as Extra-oesophageal Syndromes. The classification is as follows. 


\section{GORD is a condition which develops when the reflux of gastric content causes troublesome symptoms or complications}
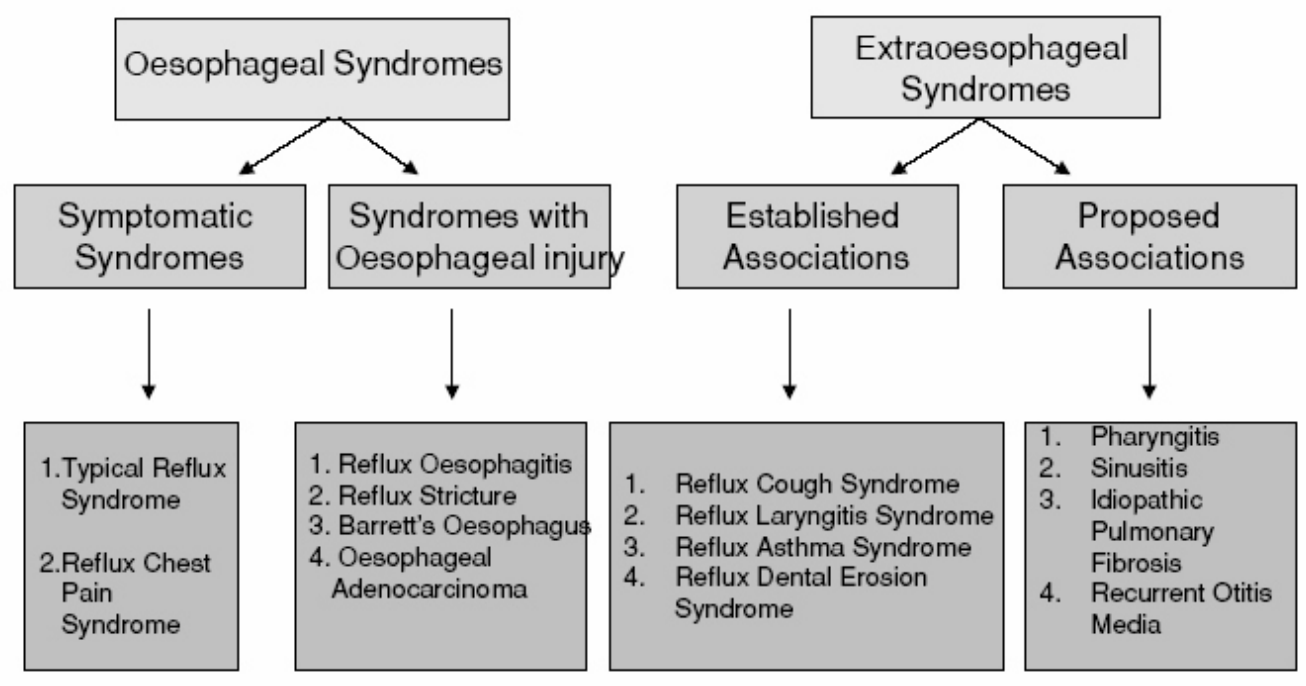

Figure 1 - The overall definition of GORD and its constituent syndromes.

\section{Symptomatic Syndromes}

Uninvestigated patients with oesophageal symptoms but without evidence of oesophageal injury are considered to have oesophageal symptomatic syndromes while patients who do have demonstrable injury are classified as oesophageal syndromes with oesophageal injury.

A patient who presents with characteristic troublesome burning chest pain and regurgitation has Typical reflux syndrome. If this type of patient undergoes endoscopy at primary care level, $>50 \%$ of the time no abnormalities would be found and it is called endoscopy negative reflux disease (ENRD). This indicates that at primary care level patients with typical reflux syndrome could be treated without subjecting them for endoscopy [2].

When a patient presents with chest pain due to oesophageal reflux without typical reflux symptoms or the pain is overshadowing typical reflux symptoms then they are classified as Reflux chest pain syndrome. The consensus report further states that Reflux chest pain syndrome is indistinguishable from ischemic cardiac pain [2]. There fore it is a diagnosis made after exclusion. This entity is identified by some as Non cardiac chest pain (NCCP). In approximately $30 \%$ of patients with recurrent NCCP, the cause is identified as GORD [3].

\section{Prevalence and Economic Burden}

Prevalence of GORD varies in different parts of the world. The prevalence is as high as $20 \%$ in some parts of Europe and North America. In Asia the incidence is low but a rising trend has been noted. In Singapore the prevalence of GORD symptoms in the general population has risen from $5.5 \%$ population in 1994 to 10.5 in 1999 [4]. It has been attributed to several causes including westernized lifestyle, better sanitation and eradication of helicobacter pylori. It is interesting to note that GORD or its complications are a rare finding in Sub Sahara Africa.

In Western countries GORD uses a significant part of the healthcare budget. It is estimated in the USA this accounts for about US $\$ 10$ billion per annum [5]. An estimated $£ 250$ million is spent every year by primary-care doctors in the UK alone on drug treatment to control the symptoms of GORD [6]. 


\section{Life style factors associated with GORD}

Lifestyle factors like overweight, obesity and smoking are associated with increased reflux symptoms [7]. This is well demonstrated in studies from Singapore which is a multiethnic country where Indians, Malays and Chinese live. Indians are more obese, eat more spicy food than their counterparts and the prevalence of GORD is also common among Indians $[8,9]$.

\section{Syndromes with oesophageal injury}

A symptomatic patient who undergoes endoscopy and has demonstrable oesophageal injury is classified under syndromes with oesophageal injury. Commonest injury identified is reflux oesophagitis which is defined endoscopically by visible breaks of the distal oesophageal mucosa [2]. The advantage of this definition is that it could easily be documented during endoscopy and provides an objective criterion for diagnosis. The consensus document had highlighted the importance of having a common classification in describing reflux oesophagitis. In this regard Los Angeles classification of reflux oesophagitis has been recommended as a simple and effective classification [10].

The characteristic symptom of a reflux stricture is persistent troublesome dysphagia. As progressive or troublesome dysphagia could be a warning symptom for cancer of the oesophagus, it warrants urgent investigation.

Barrett's oesophagus is columnar metaplasia of the lower oesophagus. It is observed that some clinicians still diagnose Barrett's oesophagus solely on the basis of endoscopic appearance. This is not a very sensitive method of diagnosing. The consensus document has suggested a new terminology. When the endoscopic appearance is suggestive of Barrett's oesophagus then it will be called Endoscopically suspected esophageal metaplasia (ESEM). Further classification will depend on the histological appearance. Only when histology confirms oesophageal metaplasia, the diagnosis of Barrett's oesophagus will be entertained. Consensus document further highlights the importance of classifying according to histological type and the extent of the lesion. Long-segment Barrett's oesophagus with intestinal type metaplasia is the most important identified risk factor for oesophageal adenocarcinoma.

Adenocarcinoma of the oesophagus is a complication of chronic GORD. Higher frequency (greater than 3 times per week) and long duration (greater than $10-20 \mathrm{yr}$ ) of GORD symptoms increases the risk of adenocarcinoma [11]. According to recent data from the United States, the incidence of adenocarcinoma of the oesophagus now has surpassed the rate of squamous carcinoma [12].

\section{Extra-oesophageal Syndromes}

There are established as well as proposed extraoesophageal manifestations of GORD. Some authorities believe extra-oesophageal manifestations are common in East and Asia. Extra-oesophageal reflux and micro aspiration may also play a part in Reflux Asthma Syndrome. It is well known that asthma treatment may predispose to GORD. GORD is usually an exacerbating co-factor rather than uniquely causative. Reflux symptoms are reported by $45 \%$ of patients with asthma compared with $10 \%$ of the general population [13]. A study has shown that asthma symptom scores continue to decline over three months period during omeprazole therapy [14]. In poorly controlled asthma it is reasonable to treat GORD as well. Chronic cough, Laryngitis and dental erosions are other well known associations of GORD. There are few other possible associations of GORD as well.

\section{Treatment of GORD}

The treatment of GORD includes lifestyle modification and standard dose proton pump inhibitors (SD PPI) fore 4 weeks. In ENRD patients, the symptoms response rate after four weeks of SD PPI therapy ranges between $46 \%$ and $57 \%$ [15]. If there is partial response to treatment then continuing for further 4 weeks could benefit. If there is no response then double 
dose proton pump inhibitors for 4 weeks is recommended. It had been shown using omeprazole $40 \mathrm{mg}$ daily for $2 / 52$ has a $37 \%$ symptom response rate in resistant cases [16]. Low doses tricyclic antidepressants or selective serotonin reuptake inhibitors have been shown to reduce pain in other functional oesophageal disorders, such as non-cardiac chest pain [17].

\section{Conclusions}

GORD is a common problem in the west with significant economic burden. Prevalence of GORD is increasing world wide. Although we lack local data, situation is not much different in Sri Lanka. The Montreal Definition and Classification of Gastro-oesophageal Reflux Disease had simplified the diagnosis. Acceptance of the definition will bring uniformity in diagnosis, treatment and research. Early detection and proper treatment of GORD could prevent potentially fatal complications.

\section{References}

1. Mark Fox, Ian Forgacs. Unexplained (non-cardiac) chest pain, Journal of the Royal College of Physicians of London; Sept/Oct. 2006, Vol 6: 445-9.

2. The Montreal definition and classification of Gastroesophageal Reflux Disease: A Global EvidenceBased Consensus, American Journal of Gastroenterology 2006; 101: 1900-20.

3. Dent, D Armstrong, Symptom evaluation in reflux disease: Workshop background, processes, terminology, recommendations, and discussion outputs, Gut 2004; 53: 1-24.

4. Lim SL, Goh WT, Lee JM, et al. Changing prevalence of gastro-esophageal reflux with changing time: Longitudinal study in an Asian population. Journal of Gastroenterology and Hepatology 2005; 20: 995-1001.
5. The burden of selected digestive diseases in the United States. Gastroenterology 2002;32: 154-6.

6. A Eggleston, A Wigerinck, S Huijghebaert, D Dubois, A Haycox. Cost effectiveness of treatment for gastrooesophageal reflux disease in clinical practice: Gut 1998; 42: 13-6.

7. M Nocon, J Labenz, SN Willich, Lifestyle Factors and Symptoms of Gastro-oesophageal reflux. A Population-Based Study. Alimentary Pharmacology and Therapeutic 2006; 23(1): 169-174.

8. National Health Surveillance Survey Singapore: Epidemiology and Disease Control Division, Ministry ofHealth, 2001.

9. KY Ho, HJ Lee, WY Wong. Curry promotes significant acid reflux in patients with gastrooesophageal reflux disease. Journal of Gastroenterology and Hepatology 2001; 16: A112.

10. Lundell et al, Gut 1999; 45: 172-180.

11. J Lagergren, R Bergstrom, A Lindgren, et al. Symptomatic gastro-oesophageal reflux as a risk factor for oesophageal adenocarcinoma. New England Journal of Medicine 1999; 340: 825-31.

12. SS Devesa SS, WJ Blot, JF Fraumeni Jr. Changing patterns in the incidence of esophageal and gastriccarcinoma in the United States. Cancer 1998; 83: 2049-53.

13. Mark Fox, Ian Forgacs, Gastro-oesophageal reflux disease, British Medical Journal 2006; 332: 88-93.

14. SM Harding, JE Richter, MR Guzzo, et al. Asthma and gastroesophageal reflux: acid suppressive therapy improves asthma outcome. American Journal of Medicine 1996; 100: 395-405.

15. T Lind, T Havelund. Heartburn without oesophagitis: efficacy of omeprazole therapy and features determining therapeutic response. Scandinavian Journal of Gastroenterology 1997; 32: 974-9.

16. BE Schenk, EJ Kuipers, EC Klinkenberg-Knol, et al. Omeprazole as a diagnostic tool in gastroesophageal reflux disease. American Journal of Gastroenterology 1997; 92: 1997-2000.

17. RO Cannon, AA Quyyumi, R Mincemoyer, et al. Imipramine in patients with chest pain despite normal coronary angiograms. New England Journal of Medicine 1994; 330: 1411-19. 